\title{
Evaluation of the anesthetic effects of MS222 in the adult Mexican axolotl (Ambystoma mexicanum)
}

\author{
This article was published in the following Dove Press journal: \\ Veterinary Medicine: Research and Reports \\ 5 January 2016 \\ Number of times this article has been viewed
}

\author{
Chiara Zullian' \\ Aurore Dodelet-Devillers' \\ Stéphane Roy ${ }^{2}$ \\ Pascal Vachon' \\ 'Département de Biomédecine \\ Vétérinaire, Faculté de Médecine \\ Vétérinaire, Université de Montréal, \\ Saint-Hyacinthe, ${ }^{2}$ Département de \\ Stomatologie, Faculté de Médecine \\ Dentaire, Montréal, Québec, Canada
}

Correspondence: Pascal Vachon Département de Biomédecine, Vétérinaire, Faculté de Médecine, Université de Montréal, Vétérinaire, 3200 Sicotte, Saint-Hyacinthe, Québec J2S 2M2, Canada Tel + I 450773852 I ext 8294 Fax +I 4507788103 Email pascal.vachon@umontreal.ca

\begin{abstract}
The Mexican axolotl (Ambystoma mexicanum) is a unique research model in several fields of medicine, where surgical and invasive procedures may be required. As yet, little is known about the efficacy of MS222 (tricaine methanesulfonate), which is the most commonly used anesthetic agent in amphibians. The main objectives of this study were to evaluate the anesthetic effects and physiological changes in adult axolotls following a 20 -minute immersion bath, containing progressive MS222 concentrations starting at $0.1 \%$. Depth of anesthesia and physiological changes were evaluated every 15 minutes post-MS222 exposure with the following parameters: righting behavior, withdrawal reflex, acetic acid test response, heart rate, and blood oxygen saturation, as well as cloacal and body surface temperatures. A 20 -minute exposure in a $0.1 \%$ MS222 immersion bath ( $\mathrm{n}=6$ animals) had no anesthetic effects on adult axolotls after 20 minutes of exposure. With a $0.2 \%$ MS222 solution, all axolotls $(n=9)$ were deeply anesthetized at 15 minutes, and $80 \%$ were still unresponsive at 30 minutes postexposure. Blood oxygen saturation and heart rate were slightly, but significantly, increased when compared with the baseline value and remained stable up to recovery. There was no significant increase in surface and cloaca temperatures, compared with baseline. With the $0.4 \%$ MS222 solution, the duration of anesthesia lasted for 90 minutes to at least 120 minutes ( $\mathrm{n}=3$ animals) and this concentration was deemed too high. In conclusion, a 20 -minute immersion bath with $0.2 \%$ MS222 may be used for short procedures (15-30 minutes) requiring anesthesia of adult axolotls.
\end{abstract}

Keywords: Ambystoma mexicanum, axolotl, amphibians, anesthesia, pain

\section{Introduction}

The Mexican axolotl is a urodele amphibian, which is extensively used as a research model due to its tissue regeneration capacities. ${ }^{1,2}$ Indeed, these are the only vertebrates that can regenerate multiple body structures following limbs, jaws, tails, skin, or spinal cord amputations or resections. ${ }^{3,4}$ They also play a unique role as models in aging diseases, oncology, and developmental biology. ${ }^{5}$ Despite the growing use of surgical models involving the Mexican axolotl, ${ }^{2,6}$ little is known about the efficacy of anesthetics in this species. MS222 (tricaine methanesulfonate) is the most commonly used anesthetic agent for amphibians, for which it was used in a variety of invasive and traumatic procedures including surgery and body marking, ${ }^{7-11}$ but rarely documented for axolotls. ${ }^{12}$ Several recent studies demonstrate the safety and efficacy of this compound used as an anesthetic bath for frogs and mole salamanders. ${ }^{13,14}$ Benzocaine and eugenol ${ }^{13,15}$ were shown to be effective alternatives to MS222; however, other drugs such as propofol ${ }^{16}$ and tiletaminezolazepam ${ }^{17}$ are not as safe and reliable for the anesthesia of amphibians. 
The primary goal of the present study was to evaluate the anesthetic effects and physiological changes in adult Mexican axolotls following three different concentrations of MS222 $(0.1 \%, 0.2 \%$, and $0.4 \%)$, when administered as a 20 -minute immersion bath. Experience with the procedures used in these experiments was previously acquired from studies performed in our laboratory, using Xenopus laevis frogs. ${ }^{14}$ To our knowledge, the present study is the first one to be conducted on the pharmacodynamic of MS222, administered as an anesthetic bath for the Mexican axolotl. However, there are a few publications available in the literature on mole salamander anesthesia in which the anesthetic properties of compounds such as benzocaine, MS222, and alfaxalone are assessed. ${ }^{13,18,19}$ Nevertheless, this species does not possess external gills, such as axolotls, which may obviously change the absorption rate of dissolved drugs. None of these studies in mole salamanders established the relationship between the anesthetic concentrations and the physiologic changes during anesthesia.

\section{Materials and methods}

\section{Animals and husbandry}

Eighteen breeding axolotls ( $\mathrm{n}=12$ females and six males; 7-8 years old), weighing between 118 and $187 \mathrm{~g}$, were used to evaluate the physiological effects of an anesthetic bath of MS222 at three different concentrations: $0.1 \%, 0.2 \%$, and $0.4 \%$. The axolotls were initially purchased from the Ambystoma Genetic Stock Center (Lexington, KY, USA) and were used to study the molecular and cellular mechanisms underlying the epimorphic regeneration in vertebrates, before being culled from the breeding colony and transferred to our protocol. Their optimal health status (no signs of disease, normal appetite, normal movements) allowed us to utilize these retired animals for our project. The protocol for the present experiment was approved by the Institutional Animal Care and Use Committee of the University of Montreal prior to animal use and in accordance with the guidelines of the Canadian Council on Animal Care. ${ }^{20}$

At the University of Montreal, breeding axolotls were housed in simple aquariums (two to three animals per tank) containing $20 \mathrm{~L}$ of a $40 \%$ modified Holtfreter's solution, purified through a carbon filter system and kept at a temperature between $18^{\circ} \mathrm{C}$ and $20^{\circ} \mathrm{C}$. To prepare $4 \mathrm{~L}$ of a stock of a modified Holtfreter's solution for use in axolotls, ${ }^{21}$ the following compounds were added: $\mathrm{KCl}(6.8 \mathrm{~g}), \mathrm{CaCl}_{2}-2 \mathrm{H}_{2} \mathrm{O}$ (14.9 g), $\mathrm{MgSO}_{4}-7 \mathrm{H}_{2} \mathrm{O}$ (37.6 g), $\mathrm{NaCl}$ (390.7 g), and Trizma (Tris(hydroxymethyl)aminomethane buffer substance) fish grade (36 g), all from Sigma-Aldrich (St Louis, MO, USA).
In order to obtain a $40 \%$ modified Holtfreter's solution, $300 \mathrm{~mL}$ of the stock solution was diluted in $20 \mathrm{~L}$ of purified water (reverse osmosis).

Animals were fed two to three times a week with soft salmon pellets (Corey Aquafeeds, Fredericton, NB, Canada) and their health status was monitored daily to exclude the presence of wounds due to fights and subsequent infections. Each aquarium was provided with hiding areas (PVC tubes) and rocks as means of environmental enrichment.

\section{MS222 solution preparation}

Solutions of $0.1 \%, 0.2 \%$, and $0.4 \%$ of MS 222 were prepared by weighing 1, 2, and $4 \mathrm{~g}$ of MS222 (Sigma-Aldrich), respectively in separate volumetric flasks and adding $1,000 \mathrm{~mL}$ of Holtfreter's solution in each flask. Sodium bicarbonate (Sigma-Aldrich) was subsequently added to each solution until a $\mathrm{pH}$ of $\sim 7$ was reached. The solution was freshly prepared and kept at room temperature prior to each use.

\section{Anesthesia methods}

A liter of the MS222 solution was transferred into a plastic clear container where axolotls were individually placed for a period of 20 minutes. The container was covered with brown paper to reduce the axolotls' light exposure during the induction phase as well as to preserve MS222 stability, which is affected by light exposure. Following the 20-minute immersion bath, axolotls were rinsed with fresh Holtfreter's solution and then placed in plastic containers filled with the same solution. Every 15 minutes, axolotls were removed from the solution and transferred on a wet surface in a dorsal decubitus to measure several physiologic parameters. During these evaluations, animals were regularly rinsed to keep their skin moist. Following the data collection, animals were returned to their original plastic containers. Nine axolotls (six females and three males; mean body weight [ \pm standard deviation] $155.6 \pm 26.2 \mathrm{~g}$ ) were first evaluated with the $0.2 \%$ MS222 concentration and then six axolotls (three males and three females; mean body weight $[ \pm$ standard deviation] 148.7.6 $\pm 31.0 \mathrm{~g}$ ) were tested at $0.1 \% \mathrm{MS} 222$ and three female axolotls (mean body weight [ \pm standard deviation] $135.3 \pm 15.5 \mathrm{~g}$ ) were tested at $0.4 \% \mathrm{MS} 222$. Only three axolotls were tested at $0.4 \%$ since the anesthesia duration was deemed too long.

\section{Evaluation of anesthesia depth and selected physiological parameters}

To evaluate the depth of anesthesia, we performed the following tests: the withdrawal reflex test, the righting reflex 
test, and the acetic acid test (AAT). The withdrawal reflex and the AAT are also used to evaluate pain in amphibians. Selected physiological parameters were also evaluated: heart rate, oxygen blood saturation, body surface temperature, and cloacal temperature. Measures were taken prior to the bath immersion (time point: -15 minutes), immediately after the immersion bath $(t=0)$, and every 15 minutes until recovery of baseline values for the AAT, withdrawal reflexes, and righting reflexes, and up to 75 and 120 minutes for the $0.2 \%$ and $0.4 \%$ MS222 baths, respectively.

The withdrawal reflex was the first parameter to be evaluated and was tested by pinching a phalangeal articulation of a hind leg with a pair of forceps, for a period of 2 seconds. The AAT is a known alternative to measure pain in nonmammalian species. ${ }^{22}$ To perform this test, a single drop $(20 \mu \mathrm{L})$ of progressive dilutions of acetic acid was placed on the skin surface of the axolotl's thigh for 2 seconds. Testing began with water $(0 \%)$, followed by the lowest concentration of acid $(5 \%)$ and proceeded with increasing concentrations $(10 \%$, $20 \%$, and $50 \%$ ) until a response (movement) was reached; such a response was interpreted as the nociceptive threshold. To prevent tissue damage, the site of the acetic acid drop was washed off with a gentle stream of Holtfreter's solution following the 2-second exposure. The righting reflex refers to the capacity of the animal to turn on its ventral surfaces, when placed on its back.

Heart rate and blood oxygen saturation were measured with a pulse oximeter (CANL-425 V, Med Associated, St Albans City, VT, USA). To assure stable readings, the probe was placed on the ventral surface of the body and a rubber band was gently applied around the body circumference to improve stability (Figure 1). The values recorded both heart rate and blood oxygen saturation and were considered reliable after stability was achieved (3-5 seconds). Body surface temperature was assessed with an infrared temperature gauge (Duratrax, Champaign, IL, USA) placed $\sim 3 \mathrm{~cm}$ above the abdominal or thoracic areas, while cloacal temperature was determined by means of a rectal thermometer (Thermalert TH-8, Physitemp, Clifton, NJ, USA), manufactured for small rodents use (mouse probe).

\section{Statistical analysis}

A repeated measures analysis of variance statistical analysis was performed with SAS (version 9.4, SAS Institute, Cary, $\mathrm{NC}$, USA) for heart rate, blood oxygen saturation, and temperature results only for animals exposed to $0.2 \%$ of MS 222 . A priori contrasts were performed for all physiological parameters compared with baselines, and then comparing the

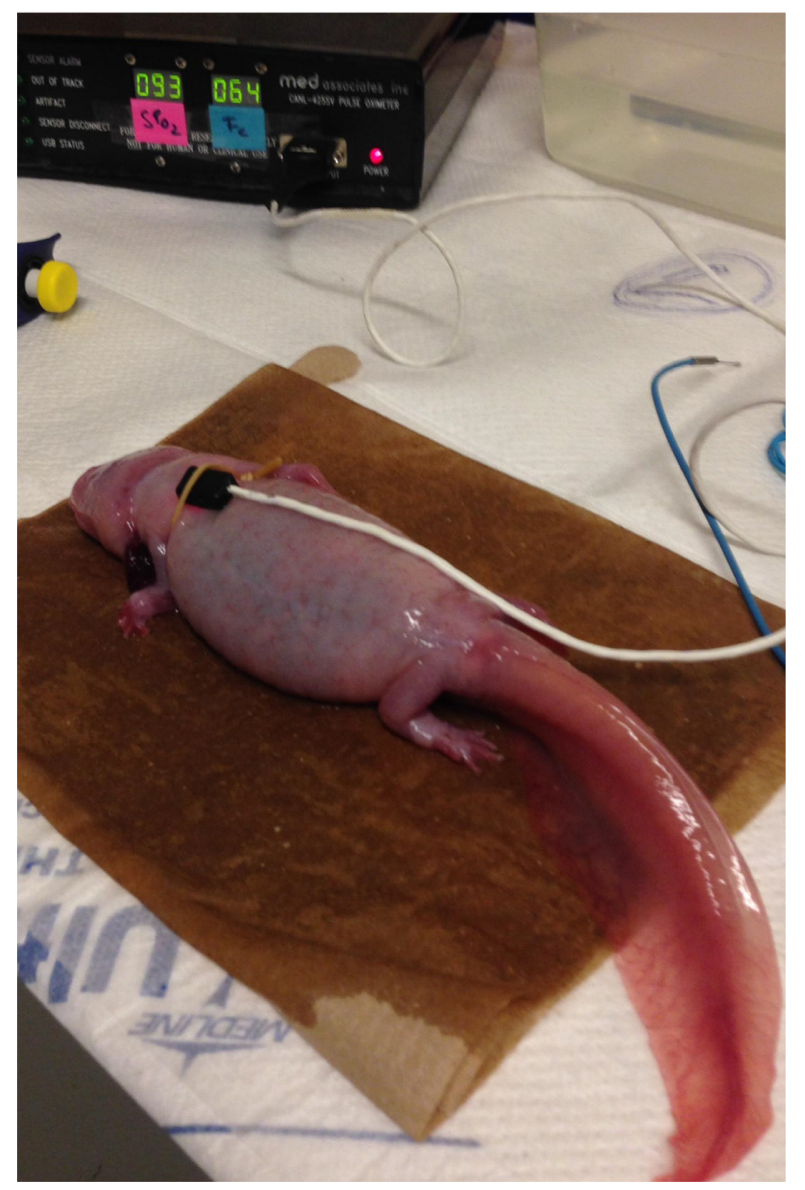

Figure I An axolotl in dorsal recumbency after a 20-minute immersion bath with MS222 (0.2\%).

Notes: Note the increased erythema of the body surface and gill darkening. A complete loss of muscular tone and absence of the righting reflex following exposures with $0.2 \%$ and $0.4 \%$ MS 222 was exhibited. Note also the position of the oximetry probe at mid-thorax (held in place with a loosely fitted elastic) used to evaluate the heart rate and the blood oxygen saturation.

first recorded data sets after baseline (time zero) to other time points following anesthesia induction to evaluate changes up to recovery. Since multiple comparisons were performed, the alpha was set for each comparison with the BenjaminiHochberg method (a default $P$-value adjustment method). ${ }^{23}$

\section{Results}

With the $0.1 \%$ MS222 solution, no anesthetic effects were observed in the six axolotls exposed for 20 minutes. After the immersion bath, the righting reflex was still present for the first 30 minutes, and for this reason, we considered that the experience reached its endpoint.

Following the $0.2 \%$ MS222 immersion bath, all axolotls ( $n=9$; six females and three females) were deeply anesthetized for 15 minutes, with a complete loss of the withdrawal reflex and a negative response to the AAT (Figure 2). Interestingly, $80 \%$ of the subjects were still unresponsive for 30 minutes 


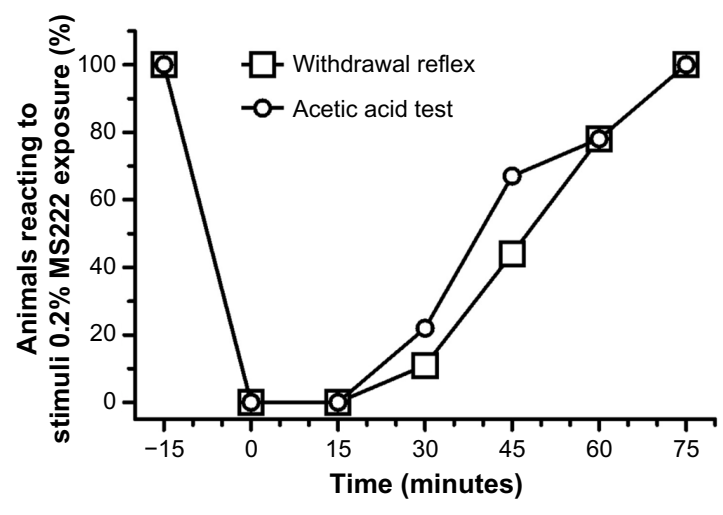

Figure 2 Percentage of adult axolotls ( $n=9$; six females and three males) reacting to the acetic acid test and withdrawal reflex following a 20 -minute $0.2 \%$ MS222 immersion bath.

Notes: -15 minutes, baseline values prior to immersion bath; time zero, time at which animals were removed from the immersion bath.

and $20 \%$ for 60 minutes. Recovery was very gradual. A complete recovery was seen in the presence of the righting reflex in all subjects at 75 minutes postimmersion bath.

The effects of $0.2 \%$ MS222 on blood oxygen saturation and heart rate are presented in Figure 3. Blood oxygen saturations did not appear to change significantly $\left(F_{5,40}=2.12\right.$, $P=$ nonsignificant) with anesthesia; however, some a priori contrasts to baseline did show significant differences at late time points. Heart rates $\left(F_{5,40}=5.08, P<0.002\right)$ increased, and a priori contrasts to baseline showed significant differences mainly at early time points. A priori contrasts during anesthesia up to recovery were nonsignificant for both heart rates and blood oxygen saturations.

The effects of $0.2 \%$ MS222 on cloacal and body surface temperatures are presented in Figure 4. Surface temperatures

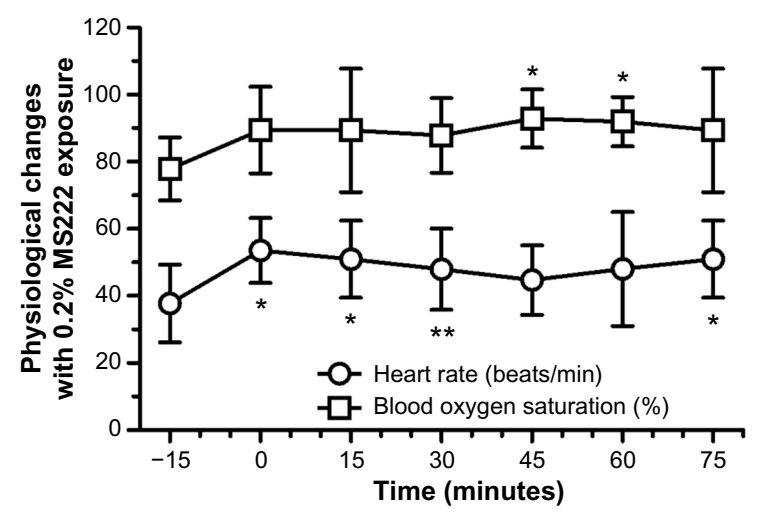

Figure 3 Heart rate (beats/min) and blood oxygen saturation (\%) (mean \pm standard deviation) of adult axolotls ( $n=9$; six females and three males) following a 20 -minute $0.2 \%$ MS222 immersion bath.

Notes: A priori comparisons to baseline showed significant differences at 0 , 15 , and 30 minutes for heart rates, and at 45 minutes and 60 minutes for blood oxygen saturation $(* P<0.01, * * P>0.05)$. All a priori contrasts during anesthesia up to recovery were nonsignificant for both parameters. -15 minutes, baseline values prior to immersion bath; time zero, time at which animals were removed from the immersion bath.

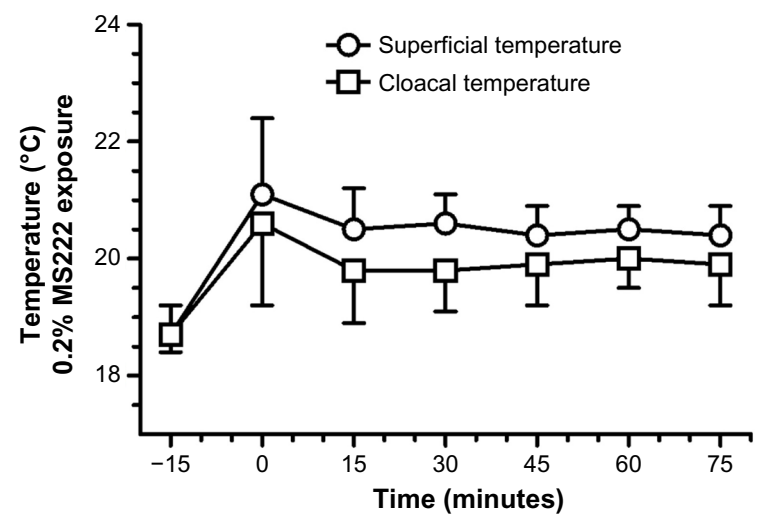

Figure 4 Cloacal and superficial temperatures $\left({ }^{\circ} \mathrm{C}\right)$ (mean \pm standard deviation) results of adult axolotls ( $n=9$; six females and three males) following a 20-minute $0.2 \%$ MS222 immersion bath.

Notes: All a priori contrasts to baseline were significant for both body surface $(P<0.000 \mathrm{I})$ and cloacal $(P<0.0 \mathrm{I})$ temperatures. A priori contrasts during anesthesia up to recovery were nonsignificant. Note that tank water temperature before the MS222 immersion bath was $18^{\circ} \mathrm{C}$. MS222 solution was kept at room temperature $\left(21^{\circ} \mathrm{C}\right) .-15$ minutes, baseline values prior to immersion bath; time zero, time at which animals were removed from the immersion bath.

significantly changed $\left(F_{5,40}=29.9, P<0.0001\right)$ with anesthesia and a priori contrasts to baseline were also significant $(P<0.0001)$. Cloacal temperatures were also significantly affected with anesthesia $\left(F_{5,40}=11.8, P<0.01\right)$, and a priori contrasts to baseline were also significant $(P<0.002)$. A priori contrasts during anesthesia up to recovery were nonsignificant for body surface and cloacal temperatures.

The $0.4 \%$ MS222 immersion bath ( $n=3$ males) caused an absence of withdrawal reflex and AAT response for the first 90 minutes and two of the three axolotls tested still had no AAT response 2 hours post-MS222 exposure. Blood oxygen saturation and heart rate did not change significantly (Figure 5). Both superficial and cloacal temperatures

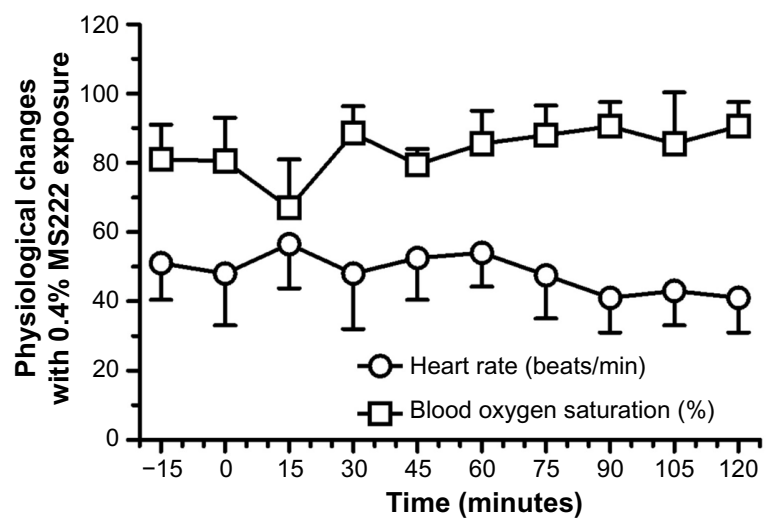

Figure 5 Heart rate (beats/min) and blood oxygen saturation (\%) (mean \pm standard deviation) of adult axolotls ( $\mathrm{n}=3$ females) following a 20 -minute $0.4 \%$ MS222 immersion bath.

Notes: -15 minutes, baseline values prior to immersion bath; time zero, time at which animals were removed from the immersion bath. 
slightly increased throughout the experiment compared with the baseline value, although findings were not statistically significant (Figure 6). On the following day, these animals had fully recovered from the anesthesia.

For all animals tested, the three different MS222 concentrations induced a change in the skin color (erythema) associated with an increase of vascularization of the body surface and darkening of the gills. With the exception of the lowest dose utilized, MS222 induced a decrease in the muscular tonus leading to a complete relaxation. On the following days, all animals recovered well from anesthesia and showed no signs of illness after the MS222 exposure. They were all reanesthetized and decapitated. Selected tissues of these animals were collected for further in vitro experiments.

\section{Discussion}

As amphibians feel pain, proper use of anesthesia must be followed in research protocols that require invasive procedures such as surgeries. ${ }^{24}$ MS222 has been commonly used for sedation, immobilization, and anesthesia of poikilothermic animals ${ }^{25-27}$ due to its anesthetic efficacy and the proven safety when administered as an immersion bath, even though other anesthetics such as benzocaine and eugenol could also be used. ${ }^{13,15}$ MS222, developed by Merck as a sulfonated analog of benzocaine, and sold by Sigma-Aldrich, has high solubility in water, which makes the drug readily available to axolotls. In particular, it is 250 times more soluble in water than benzocaine. ${ }^{28}$ MS222 has been shown to be a safer anesthetic drug in frogs than benzocaine, which causes more ventilatory failure; ${ }^{29}$ however, there is no evidence to support this in axolotls.

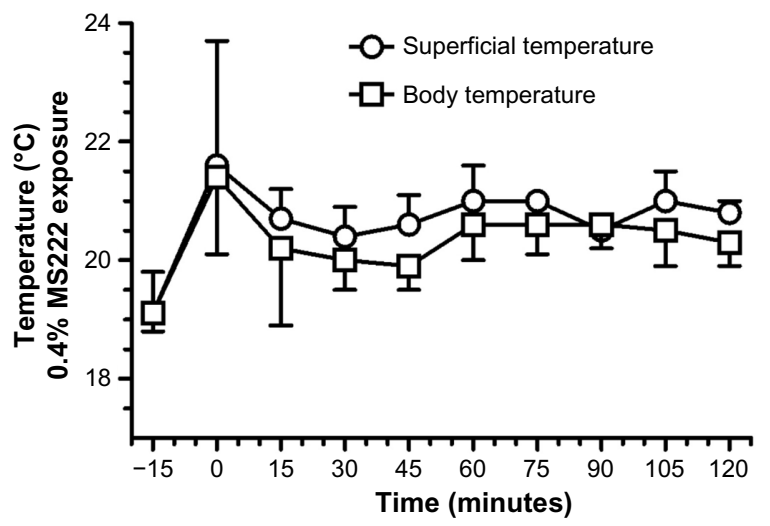

Figure $6 \mathrm{Cloacal}$ and superficial temperatures $\left({ }^{\circ} \mathrm{C}\right)$ results of adult axolotls $(n=3$ females) following a 20 -minute $0.4 \%$ MS222 immersion bath.

Notes: Note that tank water temperature before the MS222 bath immersion was $18^{\circ} \mathrm{C}$; MS222 solution was kept at room temperature $\left(21^{\circ} \mathrm{C}\right) .-15$ minutes, baseline values prior to immersion bath; time zero, time at which animals were removed from the immersion bath.
MS222 has a mechanism of action similar to other local anesthetics, which works through stabilization of cellular membranes, by inhibiting transient increases in sodium ion permeability, thereby decreasing excitability and blocking impulse conduction in excitable tissues, which may cause suppression of myocardial excitability. ${ }^{25,30}$ Increasing systemic levels of local anesthetics first block small unmyelinated fibers causing loss of sensation, then larger fibers, causing loss of motor function, followed by central nervous system depression. ${ }^{25,30}$ MS222 has been shown to cause a significant depression of electroencephalogram activity in amphibians compatible with anesthesia. ${ }^{31}$ Even though the literature offers some information on the pharmacodynamics of MS222 in several species of amphibians, little is known about its efficacy in the Mexican axolotl.

Our results showed that a 20 -minute immersion bath of $0.1 \%$ MS222 had little, if any, anesthetic effect. While this concentration has proven to be effective in younger African clawed frogs, ${ }^{14}$ in our study, axolotls tested with this concentration presented a righting reflex for the first 30 minutes following the induction bath. With $0.2 \% \mathrm{MS} 222$, axolotls were deeply anesthetized for 15 minutes and $80 \%$ of the subjects were still unresponsive for 30 minutes. With $0.4 \%$, the anesthesia lasted for 90 minutes to at least 120 minutes. Therefore, we consider that $0.2 \%$ can be an adequate choice for experimental procedures requiring short anesthesia duration, while we suggest that an intermediate concentration of $0.3 \%$ might be a more appropriate option for longer procedures; however, this needs to be evaluated in future studies. Interestingly, the righting and the withdrawal reflexes diminished and reappeared approximately at the same time after the MS222 immersion baths, following what was previously reported in X. laevis frogs. ${ }^{14}$ However, this was not seen in $X$. laevis frogs following a eugenol immersion bath, ${ }^{15}$ as the AAT reappeared earlier than the withdrawal reflex. ${ }^{28}$ Therefore, differences between amphibian species, life stages, and the use of different anesthetic compounds are to be attentively considered by the experimenter before performing any procedure requiring an anesthesia in amphibians. ${ }^{13}$

Since the use of MS222 implies less physiological variability and presents a safer therapeutic margin in amphibians, most researchers and veterinarians prefer its use to other anesthetic drugs. Our results show that even though MS222 anesthetized axolotls adequately, none of the exposed animals showed any signs of cardiorespiratory depression. This is of interest as this compound has been shown to have depressive cardiorespiratory effects in other species. ${ }^{32}$ Blood oxygen 
saturation and heart rate were increased, compared with the baseline, and remained stable throughout the anesthesia duration up to recovery. These increased physiological parameters may well be a reflection of the increase of water temperature (tank water temperature, $18^{\circ} \mathrm{C}$; water at room temperature, $21^{\circ} \mathrm{C}$ ). Similar to $X$. laevis frogs, cardiorespiratory parameters were unaffected with $0.1 \%$ and $0.2 \%$ MS222 exposures, ${ }^{14}$ suggesting that the compound had little cardiorespiratory depressive effects in amphibians.

In our study, a nonsignificant increase was observed during the anesthesia for both cloacal and superficial temperatures, when compared with baseline. Temperatures were unchanged throughout the anesthesia (up to recovery) and this is probably related to the environmental temperature. This was expected, considering that axolotls are poikilotherms.

No apparent difference between sexes was observed while evaluating data for the present study; similarly, no differences were either reported for subjects with different body sizes and when evaluating anesthetic effects and physiological changes after the anesthetic bath. This might be due in part to the homogeneity of the subjects in each group. All subjects in this study were retired breeders and their body sizes might not reflect those of nonbreeders, which are used in experimental procedures; indeed in younger animals, males may be much smaller than females, thus requiring different anesthetic concentrations and/or time of exposure. For all these reasons, it may be of interest in the future to extend the study to a bigger group of subjects, with a greater variability in body size.

When using MS222, it is always required to buffer the solution. Solutions of MS222 are acidic, due to methanesulfonic acid formation, and under these conditions, it is mostly ionized in the nonabsorbable acidic form. The low $\mathrm{pH}$ of these solutions may cause stress in animals, as well as severe apnea, hypoxia, and acidosis. ${ }^{32}$ With unbuffered solutions, the time required for induction of anesthesia is increased and the duration of anesthesia is decreased. However, in solutions buffered with sodium bicarbonate, a more rapid onset and effective anesthesia occurs, since the higher $\mathrm{pH}$ causes the amino group of MS222 to be less ionized, allowing more rapid diffusion across lipid membranes. ${ }^{29}$

In conclusion, a 20 -minute immersion bath of $0.2 \%$ MS222 may be considered for short duration, minimally invasive procedures. If longer anesthesia durations are needed, we would presently suggest an immersion bath with $0.3 \%$ MS222; however, further experiments will be necessary to confirm this assumption.

\section{Acknowledgment}

The authors would like to thank Guy Beauchamp for the statistical analyses.

\section{Disclosure}

The authors report no conflicts of interest in this work.

\section{References}

1. Lopez D, Lin L, Monaghan JR, et al. Mapping hematopoiesis in a fully regenerative vertebrate: the axolotl. Blood. 2014;124(8):1232-1241.

2. Lopez D, Scott EW. Generation of axolotl hematopoietic chimeras. J Biol Methods. 2015;2(1):e12.

3. McCusker C, Gardiner DM. The axolotl model for regeneration and aging research: a mini review. Gerontology. 2011;57:565-571.

4. Lévesque M, Gatien S, Finnson K, et al. Transforming growth factor: beta signaling is essential for limb regeneration in axolotls. PLoS One. 2007;2(11):e1227.

5. Whited JL, Lehoczky JA, Tabin CJ. Inducible genetic system for the axolotl. PNAS. 2012;8:13662-13667.

6. Khattah S, Murawala P, Andreas H, et al. Optimized axolotl (Ambystoma mexicanum) husbandry, breeding, metamorphosis, transgenesis and tamoxifen-mediated recombination. Nat Protoc. 2014;9:529-540.

7. Anholt BR, Negovetic S, Som C. Methods for anaesthetizing and marking larval anurans. Herpetol Rev. 1988;29:153-154.

8. Kaiser H, Green DM. Keeping the frogs still: Orajel ${ }^{\circledR}$ is a safe anesthetic in amphibian photography. Herpetol Rev. 2001;32:93-94.

9. Kaplan HM. Anesthesia in amphibians and reptiles. Fed Proc. 1969;28: 1541-1546.

10. Stevens CW. Analgesia in amphibians: preclinical studies and clinical applications. Clin North Am Exot Anim Pract. 2011;14:33-44.

11. Wright KM. Amphibian husbandry and medicine In: Mader DR, editor. Reptile Medicine and Surgery. Philadelphia, PA: WB Saunders; 1996:436-458.

12. Menger B, Vogt PM, Jacobsen ID, et al. Resection of a large intraabdominal tumor in the Mexican axolotl: a case report. Vet Surg. 2010;39:232-233.

13. Cecala KK, Steven JP, Dorcas ME. A comparison of the effectiveness of recommended doses of MS 222 (tricaine methanesulfonate) and Orajel (benzocaine) for amphibian anesthesia. Herpetol Rev. 2007;38: 63-66.

14. Lalonde-Robert V, Beaudry F, Vachon P. Pharmacologic parameters of MS222 and physiologic changes in frogs (Xenopus laevis) after immersion at anesthetic doses. J Am Assoc Lab Anim Sci. 2012;51: 464-468.

15. Guénette SA, Hélie P, Beaudry F, Vachon P. Eugenol for anesthesia of Xenopus laevis frogs. Vet Anaesth Analg. 2007;34:164-170.

16. Guénette SA, Hélie P, Beaudry F, Vachon P. Anesthetic properties of propofol in African clawed frogs (Xenopus laevis). J Am Assoc Lab Anim Sci. 2008;47:35-38.

17. Letcher J, Durante R. Evaluation of use of tiletamine-zolazepam for anesthesia of leopard frogs, Rana pipiens. J Am Vet Med Assoc. 1995;207:80-82.

18. McMillan MW, Leece EA. Immersion and branchial/transcutaneous irrigation anaesthesia with alfaxalone in a Mexican axolotl. Vet Anaesth Analg. 2011;38:619-623.

19. Vanable JJ. Benzocaine: an excellent amphibian anesthetic. Axolotl Newslett. 1985;14:19-21.

20. Canadian Council on Animal Care. Guide to the Care and Use of Experimental Animals. Vols 1 and 2. Ottawa, Canada; Canadian Council on Animal Care; 1993.

21. Wright KM, Whitaker BR. Pharmacotherapeutics. In: Wright KM, Whitaker BR, editors. Amphibian Medicine and Captive Husbandry. Malabar: Krieger Publishing; 2001:309-330.

22. Stevens CW. Alternative to the use of mammals for pain research. Life Sci. 1992;50:901-912. 
23. McDonald JH. Multiple comparisons. In: Handbook of Biological Statistics. 3rd ed. Baltimore, MD: Sparky House Publishing; 2014: 254-260.

24. Guénette SA, Giroux MC, Vachon P. Pain perception and anesthesia in research frogs. Exp Anim. 2013;62:87-92.

25. Machin KL. Amphibian pain and analgesia. J Zoo Wild Med. 1999;30: 2-10.

26. Martinez M, Hedrick MS. Cardiorespiratory control in paralyzed, decerebrate toads (Bufo marinus): influence of anesthesia and postsurgical recovery time. Comp Biochem Physiol. 2002;132A:S27-S36.

27. Sladky KK, Swanson CR, Stoskopf MK, Loomis MR, Lewbart GA. Comparative efficacy of tricaine methane-sulfonate and clove oil for use as anesthetics in red pacu (Piaractus brachypomus). Am JVet Res. 2001;62:337-342.
28. Herr VD, Sonnenburg DC, Courogan PM, Filamengo SA, Downes H. Muscle weakness during tricaine anesthesia. Comp Biochem Physiol. 1995;110C:289-296.

29. CakirY, Strauch SM. Tricaine (MS-222) is a safe anesthetic compound compared to benzocaine and pentobarbital to induce anesthesia in leopard frogs (Rana pipiens). Pharmacol Rep. 2005;57:467-474.

30. Butterworth JF, Strichartz GR. Molecular mechanism of local anesthesia: a review. Anesthesiology. 1990;72:711-734.

31. Lalonde-Robert V, Desgent S, Duss S, Vachon P. Electroencephalographic and physiologic changes during tricaine methanesulfonate euthanasia in Xenopus laevis frogs. J Am Assoc Lab Anim Sci. 2012;51:622-627.

32. Downes U. Tricaine methanesulfonate in amphibians: a review. Bull Assoc Rept Amph Vet. 1995;5:11-16.

\section{Publish your work in this journal}

Veterinary Medicine: Research and Reports is an international, peer-reviewed, open access journal publishing original research, case reports, editorials, reviews and commentaries on all areas of veterinary medicine. The manuscript management system is completely online and includes a very quick and fair peer-review system.
Visit http://www.dovepress.com/testimonials.php to read real quotes from published authors. 